\title{
Prediction of pressure-induced stabilization of noble-gas-atom compounds with alkali oxides and alkali sulfides
}

\author{
Hao Gao, ${ }^{1}$ Jian Sun, ${ }^{1, \text { * }}$ Chris J. Pickard, ${ }^{2,3}$ and Richard J. Needs ${ }^{4}$ \\ ${ }^{1}$ National Laboratory of Solid State Microstructures, \\ School of Physics and Collaborative Innovation Center of Advanced \\ Microstructures, Nanjing University, Nanjing 210093, China \\ ${ }^{2}$ Department of Materials Science \& Metallurgy, University of Cambridge, \\ 27 Charles Babbage Road, Cambridge CB3 OFS, UK \\ ${ }^{3}$ Advanced Institute for Materials Research, Tohoku University, 2-1-1 Katahira, Aoba, Sendai, 980-85r7, Japan \\ ${ }^{4}$ Theory of Condensed Matter Group, Cavendish Laboratory, \\ J J Thomson Avenue, Cambridge CB3 OHE, UK
}

(Dated: January 15, 2019)

\begin{abstract}
The cubic antifluorite structure comprises a face-centered cubic sublattice of anions with cations on the tetrahedral sites. The voids in the antifluorite structure that are crucial for superionicity in $\mathrm{Li}_{2} \mathrm{O}$ might also act as atomic traps. Trapping of guest atoms and small molecules within voids of a host structure leads to the formation of what are known as clathrate compounds. Here we investigate the possibility of trapping helium or larger neon guest atoms under pressure within alkali metal oxide and sulfide structures. We find stable helium and neon-bearing compounds at very low pressures. These structures are stabilized by a reduction in volume from incorporation of helium or neon atoms within the antifluorite structure. We predict that $\mathrm{NeCs}_{2} \mathrm{~S}$ could be stable at ambient pressure. Our study suggests a novel class of alkali oxide and sulfide materials incorporating noble gas atoms that might potentially be useful for gas storage.
\end{abstract}

\section{INTRODUCTION}

Alkali metal oxides are used in catalysing reactions $\stackrel{1}{1}$, capture and storage of $\mathrm{CO}_{2}{ }_{2}^{2}$, gas detectors, and for lowering the work function of photo-cathodes ${ }^{3}$. Lithium oxide $\left(\mathrm{Li}_{2} \mathrm{O}\right)$ is a key battery material, and it is also used within the nuclear industry. At low pressures alkali oxides ${ }^{4-13}$ and sulfides $14-19$ normally crystallize in the antifluorite structure of space group $F m \overline{3} m$, which contains octahedral voids, see Fig. 1. $\mathrm{Li}_{2} \mathrm{O}$ exhibits superionic conductivity at temperatures above $1200 \mathrm{~K}$ in which diffusing $\mathrm{Li}^{+}$ions carry electrical current by hopping from one void to another, while the oxygen atoms remain within a rigid framework ${ }^{20,21}$. The voids in the antifluorite structure that are crucial for superionicity in $\mathrm{Li}_{2} \mathrm{O}$ might also act as atomic traps. Trapping of guest atoms and small molecules within voids of a host structure can lead to the formation of what are known as clathrate compounds $\underline{22}$. Clathrates involving many different guest species have been observed, such as clathrate hydrates with inclusion of helium $(\mathrm{He})$, neon $(\mathrm{Ne})$, argon $(\mathrm{Ar})^{23}-25$, or other noble gas atoms. Trapped noble gas atoms have also been found in alkali metals 26 and oxides 27.28 under pressure. Such compounds have been reviewed by Grochala 29 .

Here we investigate the possibility of trapping He or larger Ne guest atoms under pressure within alkali metal oxide and sulfide structures. By studying different noble gas atoms, alkali oxides and sulfides, we are able to assess the effects of changing both the host and guest species. At zero pressure the thermodynamic driving force for including $\mathrm{He}$ or Ne atoms within the alkali oxides or sulfides is small. The inertness of $\mathrm{He}$ and Ne suggests that they might be included within structures containing voids without substantial changes in the host structure. Struc-

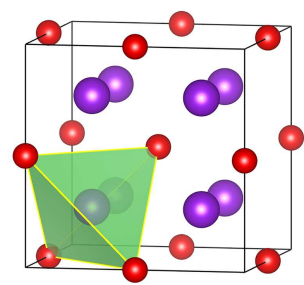

(a)

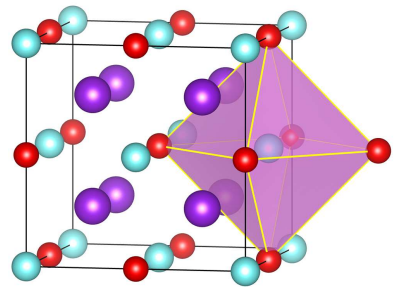

(b)
FIG. 1. Crystal structure of antifluorite alkali metal oxide with and without helium atoms. (a) The antifluorite structure of $\mathrm{Na}_{2} \mathrm{O}$. The $\mathrm{Na}$ atoms are shown in purple and oxygen atoms in red. The $\mathrm{Na}$ atoms occupy tetrahedral positions, and one of the $\mathrm{Na}$ atoms is shown at the center of its green tetrahedron. (b) He atoms (light blue) are included within the octahedral voids, and one of the octahedrons is highlighted in purple with a $\mathrm{He}$ atom visible at its center.

tures containing voids are unlikely to be stable at high pressures, and it is more likely that the thermodynamically stable state at high pressures of, say, a mixture of $\mathrm{He}$ and $\mathrm{Li}_{2} \mathrm{O}$, will consist of hexagonal-close-packed (hcp) $\mathrm{He}$ and a dense high-pressure phase of $\mathrm{Li}_{2} \mathrm{O}$. The energetic stability of structures consisting of $\mathrm{He}$ or $\mathrm{Ne}$ atoms trapped in the antifluorite structures of alkali oxides and sulfides therefore rests on a delicate balance of competing effects.

The ambient and high pressure structures of alkali metal sulfides have been studied extensively, and good agreement between experiment and the results of firstprinciples density-functional-theory (DFT) calculations has been found $\stackrel{8}{ }$. There is also good agreement between 
TABLE I. Lattice constant $(\AA)$ of $\mathrm{K}_{2} \mathrm{O}$ with different exchange-correlation functionals and $\mathrm{K}$ pseudopotentials.

\begin{tabular}{ccc}
\hline \hline \multirow{2}{*}{$\begin{array}{c}\text { Exchange-Correlation } \\
\text { Functionals }\end{array}$} & \multicolumn{2}{c}{ Valence electrons } \\
\cline { 2 - 3 } optB88-vdW $^{30}$ & 6.435 & $3 s^{2} 3 p^{6} 4 s^{1}$ \\
optB86b-vdW $^{31}$ & 6.432 & 6.382 \\
vdW-DF2 ${ }^{32}$ & 6.557 & 6.373 \\
DFT-D2 $^{33}$ & 6.452 & 6.518 \\
DFT-D3 & 6.494 & 6.405 \\
LDA & 6.272 & 6.436 \\
PBE & 6.537 & 6.488 \\
PBEsol & 6.413 & 6.487 \\
\hline Experiment $(317 \mathrm{~K})^{36}$ & \multicolumn{2}{c}{6.436} \\
\hline \hline
\end{tabular}

experiment and DFT results for alkali metal oxides under ambient conditions, although their high-pressure behavior has not been studied in depth experimentally, except for $\mathrm{Li}_{2} \mathrm{O}^{7,9}$.

A transition from the antifluorite to an orthorhombic anticotunnite structure of Pnma symmetry has been observed in $\mathrm{Li}_{2} \mathrm{O}$ at pressures around $50 \mathrm{GPa}^{7.9}$. Transitions from the antifluorite to anticotunnite structures have also been reported in $\mathrm{Li}_{2} \mathrm{~S}$ at $12 \mathrm{GPa}^{15}, \mathrm{Na}_{2} \mathrm{~S}$ at $7 \mathrm{GPa}^{16}$ and in $\mathrm{Rb}_{2} \mathrm{~S}$ below $0.7 \mathrm{GPa}^{18}$. Interestingly, the anticotunnite structure has not been observed in $\mathrm{K}_{2} \mathrm{~S}$, and a distorted $\mathrm{Ni}_{2}$ In-type structure of space group $P 6_{3} / m m c$ has instead been found at $6 \mathrm{GPa}^{17}$. A transition from the antifluorite to anticotunnite structure has been observed in $\mathrm{Rb}_{2} \mathrm{~S}$ below $0.7 \mathrm{GPa}$, followed by a transition to a $\mathrm{Ni}_{2}$ In-type structure at $2.6 \mathrm{GPa}^{18}$. $\mathrm{Cs}_{2} \mathrm{~S}$ has been reported to crystallize in the anticotunnite structure ${ }^{14}$ under ambient conditions, and to transform into a distorted $\mathrm{Ni}_{2} \mathrm{In}$-type structure by about $5 \mathrm{GPa}^{19}$.

\section{METHODS}

We used the $a b$ initio random structure searching (AIRSS) method and the CASTEP plane-wave DFT code 40 to find stable structures of alkali oxides and sulfides containing $\mathrm{He}$ or $\mathrm{Ne}$ atoms. In the AIRSS approach randomly chosen structures are relaxed to local minima in the enthalpy $41-43$. AIRSS has been successfully applied to many systems, including alkali metals 44 , and various oxides such as carbon monoxide ${ }^{45,46}$. The electronic structures and enthalpies were calculated using the CAsteP code. Very similar results were obtained with the projector augmented wave (PAW) method implemented in the Vienna $a b$ initio simulation package (VASP) 47 .

We performed AIRSS calculations for a variety of
TABLE II. Lattice constant $(\AA)$ of HCP He (18MPa)

\begin{tabular}{ccc}
\hline \hline & \multicolumn{2}{c}{ Lattice constant } \\
\cline { 2 - 3 } Exchange-Correlation & $\mathrm{a}$ & $\mathrm{c}$ \\
Functionals & 2.885 & 4.756 \\
optB88-vdW & 2.712 & 4.444 \\
DFT-D3 & 2.426 & 3.988 \\
LDA & 2.864 & 4.699 \\
PBE & 3.016 & 4.948 \\
PBEsol & 3.460 & 5.600 \\
\hline Experiment(3.3K) $)^{37}$ &
\end{tabular}

TABLE III. Lattice constant $(\AA)$ of FCC Ne

\begin{tabular}{ccc}
\hline \hline & \multicolumn{2}{c}{ Pressures } \\
\cline { 2 - 3 } Exchange-Correlation & 0 & $10 \mathrm{GPa}$ \\
Functionals & 4.264 & 3.560 \\
optB88-vdW & 4.345 & 3.601 \\
DFT-D3 & 3.852 & 3.442 \\
LDA & 4.510 & 3.619 \\
PBE & 4.560 & 3.563 \\
PBEsol & $4.42(4.2 \mathrm{~K})^{38}$ & $3.567(300 \mathrm{~K})^{39}$ \\
\hline Experiment
\end{tabular}

$\mathrm{Na} / \mathrm{O} / \mathrm{He}$ stoichiometries at a pressure of $15 \mathrm{GPa}$. The most stable $\mathrm{Na} / \mathrm{O}$ compound at $15 \mathrm{GPa}$ was found to be $\mathrm{Na}_{2} \mathrm{O}$ in the antifluorite structure, as expected, and we found the anticotunnite structure and a $\mathrm{Ni}_{2} \mathrm{In}$-type structure of space group $P 6_{3} / m m c$. We also found a structure of chemical composition $\mathrm{HeNa}_{2} \mathrm{O}$ in which $\mathrm{Na}_{2} \mathrm{O}$ adopts the antifluorite structure and the $\mathrm{He}$ atoms sit at the octahedral voids, so that the $F m \overline{3} m$ symmetry of the antifluorite structure is maintained. The $\mathrm{HeNa}_{2} \mathrm{O}$ structure was found to be slightly more stable than antifluorite $\mathrm{Na}_{2} \mathrm{O}$ at $15 \mathrm{GPa}$. We proceeded to investigate the possibility of stable antifluorite structures of alkali oxides and sulfides with $\mathrm{He}$ or $\mathrm{Ne}$ atoms placed at the octahedral voids. We then widened our study to include other competitive structures of the alkali oxides and sulfides. As shown in TABLE I, II and III, we have calculated the cell parameters of $\mathrm{K}_{2} \mathrm{O}$, He and Ne with several different exchange-correlation functionals and pseudopotentials, and compared them with experimental data. The results demonstrate the necessity of using pseudopotentials with nine valence electrons for alkali metal elements. The lattice parameters of solid He calculated by DFT is about $10 \%$ lower than in the experimental data. The deviations are mainly attributed to nuclear quantum effects and zero-point energy. Other ab initio methods, such as Diffusion quantum Monte Carlo (DMC) can be performed to obtain accurate results in excellent agree- 
ment with experiment $\underline{48,49}$. Grimme's dispersion corrected (DFT-D) methods $\frac{33,34}{34}$ perform very well in this test; however, the parameters were fitted to experimental data at ambient pressure. Therefore they may not be suitable for calculations at high pressures. The vdWDF corrections of Lundqvist et $a l^{50}$ have a non-local term that is dependent on the electron density, which should be more appropriate than DFT-D methods at high pressure. Therefore we have used the optB88-vdW functional ${ }^{30}$ and pseudopotentials with 9 valence electrons for alkali metals in our plane-wave calculations. A basis set energy cutoff of $800 \mathrm{eV}$ was used, except for $\mathrm{Na}$ compounds, in which a higher cutoff energy of 980 $\mathrm{eV}$ was used. The Brillouin zone was sampled using a $k$ point grid spacing of $2 \pi \times 0.02 \AA^{-1}$ for the final converged results reported in this paper. We have also performed calculations using Heyd-Scuseria-Ernzerhof (HSE06) hybrid functionals $\frac{51}{5}$ wich provide accurate electronic band gaps for $\mathrm{Na}_{2} \mathrm{O}$ and $\mathrm{HeNa}_{2} \mathrm{O}$ when using pseudopotentials with 7 valence electrons.

\section{RESULTS AND DISCUSSION}

As well as the antifluorite and anticotunnite structures, we considered the $\mathrm{CdCl}_{2}(R \overline{3} m), \gamma \mathrm{US}_{2}(P \overline{6} 2 m)$, and $\mathrm{CaCl}_{2}(P n n m)$ structures of the oxides, and Pnnm, $P m m a$ and the $P 6_{3} / m m c$ structures of the alkali metal sulfides. We found that the enthalpy-pressure curves of the Pmma phase reported for $\mathrm{K}_{2} \mathrm{~S}$ in Ref. 17 were very close to those of the $P 6_{3} / m m c$ structure for the sulfides from $\mathrm{Li}_{2} \mathrm{~S}$ to $\mathrm{Cs}_{2} \mathrm{~S}$ at the pressures studied, and therefore we have not reported results for the Pmma structure. The energetically competitive structures were relaxed, and their relative enthalpies are reported in Fig. 2. Interestingly, we found that the transition from the antifluorite to anticotunnite structure in $\mathrm{Na}_{2} \mathrm{O}$ occurs at fairly low pressures, but at higher pressures a transition back to antifluorite occurs with included He atoms. Further details of the phase transition pressures are reported in the Supplemental Material ${ }^{52}$.

Consider a static lattice calculation of the total enthalpy $H=U+p V$, where $U$ is the total internal energy, $p$ is the pressure and $V$ is the volume. Taking $\mathrm{Na}_{2} \mathrm{O}+\mathrm{He}$ as an example, the reduction in the total volume on forming $\mathrm{HeNa}_{2} \mathrm{O}(F m \overline{3} m)$ at $20 \mathrm{GPa}$ corresponds to about $1 / 4$ of the volume of a He atom in hcp He at the same pressure. We find that the formation of $\mathrm{HeNa}_{2} \mathrm{O}(F m \overline{3} m)+$ hcp He from $\mathrm{He}+\mathrm{Na}_{2} \mathrm{O}(F m \overline{3} m)$ increases the total internal energy by $244 \mathrm{meV}$ per formula unit, but the $p V$ term decreases by $486 \mathrm{meV}$ per formula unit. This shows that although the interactions between the He atoms and the host antifluorite $\mathrm{Na}_{2} \mathrm{O}$ are not energetically favorable, the reduction in volume from inclusion of the He atoms lowers the overall enthalpy. The pressure derivative of the enthalpy is equal to the volume $(d H / d p=V)$, so that the slopes of the curves in Fig. 2(b) give the volumes relative to the reference $\mathrm{HeNa}_{2} \mathrm{O}(F m \overline{3} m$ ) phase (denoted by the dashed-dotted line). The most stable form at low pressures is $\mathrm{Na}_{2} \mathrm{O}(F m \overline{3} m)+$ hcp He, which transforms into $\mathrm{Na}_{2} \mathrm{O}($ Pnma $)+$ hep He at $9.3 \mathrm{GPa}$. At $13 \mathrm{GPa}$ a transformation occurs to $\mathrm{HeNa}_{2} \mathrm{O}(F m \overline{3} m)$, which is stable up to just above $100 \mathrm{GPa}$. We observe from the slopes of the enthalpy-pressure curves in Fig. 2(b) that at $13 \mathrm{GPa}$ the volume of $\mathrm{HeNa}_{2} \mathrm{O}(F m \overline{3} m)$ is smaller than that of the other $\mathrm{Na}_{2} \mathrm{O}+\mathrm{He}$ phases. The thermodynamic driving force for the initial incorporation of $\mathrm{He}$ atoms is therefore the associated reduction in total volume. A maximum in the enthalpy reduction from $\mathrm{He}$ inclusion in $\mathrm{Na}_{2} \mathrm{O}$ occurs at about $40 \mathrm{GPa}$. At higher pressures the stability of the inclusion compound is reduced because the voids in the compressed antifluorite structure are no longer large enough to accommodate the He atoms, which results in an increase in the internal energy, and $\mathrm{HeNa}_{2} \mathrm{O}(F m \overline{3} m)$ eventually becomes unstable to formation of the $P 6_{3} / m m c$ structure just above 100 GPa, see Fig. 2 .

The transition pressures for noble gas inclusion are lower for the sulfides than the oxides because the larger size of the $\mathrm{S}$ atom leads to larger octahedral voids in the sulfide structures. There is an upper pressure limit to the stability of each of the noble gas compounds discussed here, which depends on the sizes and chemical identities of the host and guest species. The larger stabilization enthalpies of $\mathrm{Ne}$ atoms in alkali sulfides compared with $\mathrm{He}$ is due to the better matching of the size of the Ne atom with that of the voids in the antifluorite sulfide structures. More $p V$ energy is gained by including a Ne atom within the antifluorite structure rather than a smaller $\mathrm{He}$ atom. If the void is large enough the additional strain energy from including the larger $\mathrm{Ne}$ atom is small, and it is energetically favorable to include the Ne atom. In other words, the stabilization enthalpy is largest at pressures for which the sizes of the voids and included alkali atoms are well matched.

We find ranges of stability for antifluorite $\mathrm{HeK}_{2} \mathrm{O}$ and $\mathrm{HeRb}_{2} \mathrm{O}$, as well as $\mathrm{HeNa}_{2} \mathrm{O}$, but $\mathrm{HeLi}_{2} \mathrm{O}$ and $\mathrm{HeCs}_{2} \mathrm{O}$ are thermodynamically unstable at the pressures studied, see Fig. 2(a-e). The stability ranges are 13-105 GPa $\left(\mathrm{HeNa}_{2} \mathrm{O}\right), 3-45 \mathrm{GPa}\left(\mathrm{HeK}_{2} \mathrm{O}\right)$, and 3.7-34.5 GPa $\left(\mathrm{HeRb}_{2} \mathrm{O}\right)$. Replacing the $\mathrm{O}$ atoms by larger $\mathrm{S}$ atoms we find that $\mathrm{HeLi}_{2} \mathrm{~S}, \mathrm{HeNa}_{2} \mathrm{~S}$ and $\mathrm{HeCs}_{2} \mathrm{~S}$ are not thermodynamically stable, but that $\mathrm{HeK}_{2} \mathrm{~S}$ and $\mathrm{HeRb}_{2} \mathrm{~S}$ have small regions of stability at low pressures with small stabilization enthalpies, and $\mathrm{HeRb}_{2} \mathrm{~S}$ is predicted to be stable at ambient pressure. The stability ranges are $1.3-2.4 \mathrm{GPa}$ $\left(\mathrm{HeK}_{2} \mathrm{~S}\right)$ and $0-2 \mathrm{GPa}\left(\mathrm{HeRb}_{2} \mathrm{~S}\right)$, see Fig. 2(f-j). The inclusion of He atoms within the alkali sulfides is therefore not particularly favorable. Replacing the He atoms by larger $\mathrm{Ne}$ atoms, we find that $\mathrm{NeNa}_{2} \mathrm{~S}, \mathrm{NeK}_{2} \mathrm{~S}, \mathrm{NeRb}_{2} \mathrm{~S}$, and $\mathrm{NeCs}_{2} \mathrm{~S}$ are predicted to be stable under ambient or high pressure, but $\mathrm{NeLi}_{2} \mathrm{~S}$ is unstable up to $20 \mathrm{GPa}$. The stability ranges are also larger: $8.8-13 \mathrm{GPa}\left(\mathrm{NeNa}_{2} \mathrm{~S}\right)$, 0-7.5 GPa $\left(\mathrm{NeK}_{2} \mathrm{~S}\right), 0-6 \mathrm{GPa}\left(\mathrm{NeRb}_{2} \mathrm{~S}\right)$, and 0-3.3 Ga $\left(\mathrm{NeCs}_{2} \mathrm{~S}\right)$, see Fig. $2(\mathrm{k}-\mathrm{O})$. The maximum stabilization energies for the helium alkali oxides, helium alkali sul- 


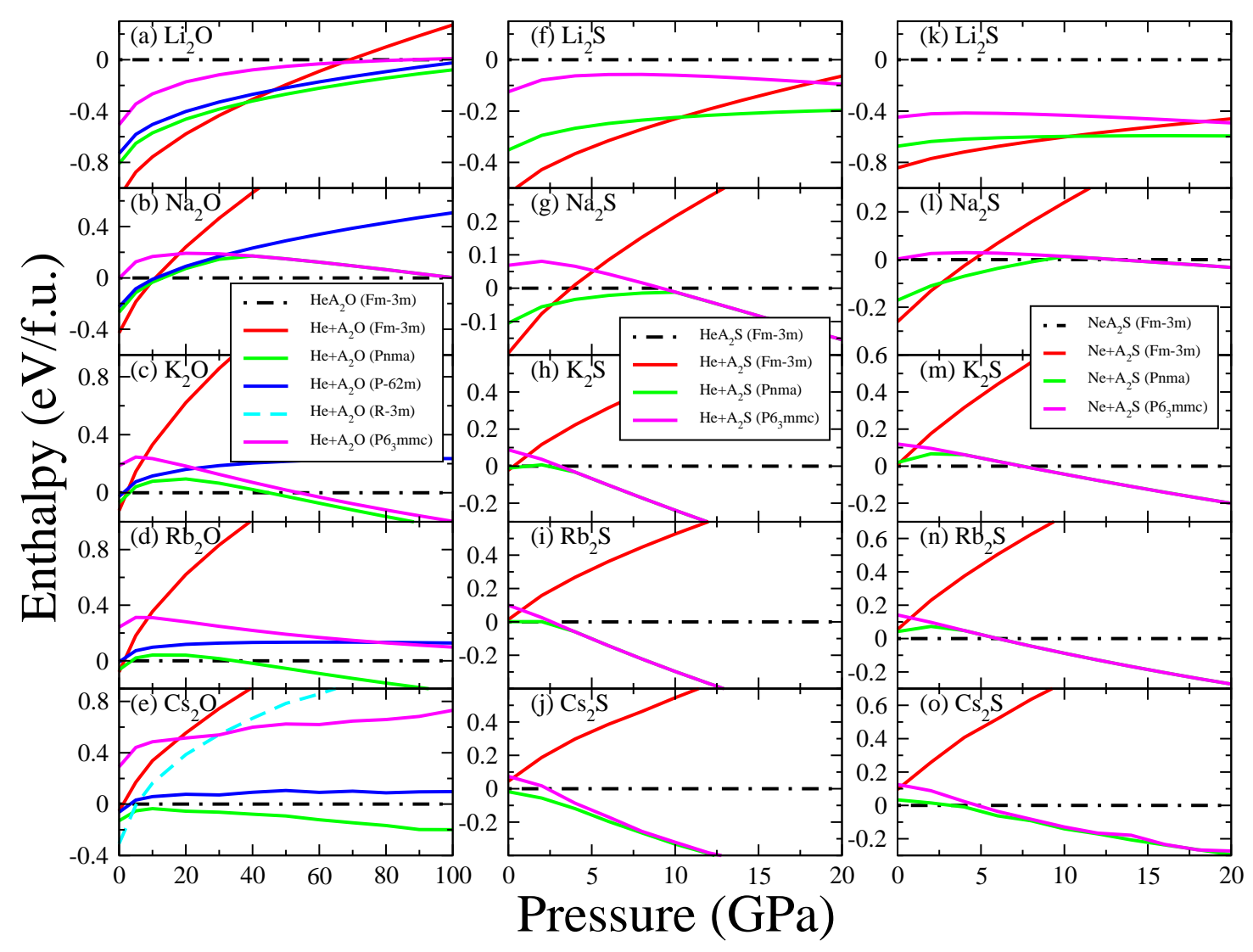

FIG. 2. Enthalpy-pressure relations for noble gas compounds. (a-e) He in alkali metal oxides, (f-j) He in alkali metal sulfides, and $(\mathrm{k}-\mathrm{O}) \mathrm{Ne}$ in alkali metal sulfides. The enthalpies are given with respect to those of the alkali oxides/sulfides with included noble gas atoms.

fides, and neon alkali sulfides are: $172 \mathrm{meV}\left(\mathrm{HeNa}_{2} \mathrm{O}\right.$ at $40 \mathrm{GPa}) ; 8 \mathrm{meV}\left(\mathrm{HeK}_{2} \mathrm{~S}\right.$ at $\left.2 \mathrm{GPa}\right)$; and $72 \mathrm{meV}\left(\mathrm{NeRb}_{2} \mathrm{~S}\right.$ at $2 \mathrm{GPa}$ ).

To validate the stability of the noble gas ternary compounds at low pressures we calculated enthalpy-pressure relations in the range $0-5 \mathrm{GPa}$ using the PBE, PBEsol ${ }^{35}$ and DFT-D $3^{34}$ functionals. As can be seen in Fig. 3, including the $\mathrm{vdW}$ interactions reduces the formation pressures of noble gas compounds. Different vdW correction methods, such as optB88-vdW 30 and DFT-D3 ${ }^{34}$, give similar results. We predict that $\mathrm{NeK}_{2} \mathrm{~S}, \mathrm{NeRb}_{2} \mathrm{~S}$ and $\mathrm{NeCs}_{2} \mathrm{~S}$ could exist at ambient pressure.

Each of the materials investigated is an insulator. The bandstructures of the alkali oxides and sulfides consist of narrow and rather widely spaced bands, as shown in Fig. 4 for $\mathrm{Na}_{2} \mathrm{O}$ at $20 \mathrm{GPa}$. The orbitals at the valence band maximum arise mainly from the $\mathrm{O} 2 p$ levels, which capture the valence $3 s$ electrons from the $\mathrm{Na}$ atoms. The bands at about $-14.5 \mathrm{eV}$ arise from the $\mathrm{O} 2 s$ levels, and those at $-22.0 \mathrm{eV}$ arise from the $\mathrm{Na} 2 p$ levels. Additional $\mathrm{H}$ atoms occupy a band at about $-13.5 \mathrm{eV}$ which shows a small amount of hybridization with the O $2 s$ levels. The $\mathrm{Na} 2 s$ levels lie at about $-50 \mathrm{eV}$ (not shown).

In $\mathrm{K}_{2} \mathrm{~S}$ at $2 \mathrm{GPa}$ the $\mathrm{O} 2 p$ levels capture the $\mathrm{K} 4 s$ electrons, while the $\mathrm{O} 2 s$ levels lie at about $-9.4 \mathrm{eV}$. The $\mathrm{K} 3 p$ levels lie at about $-12.6 \mathrm{eV}$. When He atoms are included they occupy a $1 s$ band which is very close in energy to the $\mathrm{K} 3 p$ levels, and there is considerable hybridization between these orbitals, see the Supplemental Material ${ }^{52}$. If $\mathrm{Ne}$ atoms are included instead of $\mathrm{He}$, we find that the Ne $2 p$ levels sit at an energy of about $-10.0 \mathrm{eV}$, just below the O $2 s$ levels.

The inclusion of noble gas atoms does not in general alter the oxygen, sulfur or alkali-metal-derived bands very much, which is consistent with the interaction between the noble gas atoms and the host oxide/sulfide being fairly weak. However, in some cases significant hybridization between the alkali atom and O $2 s$ levels can occur. Bandstructures for other oxides and sulfides, with and without included He and Ne atoms are shown in the Supplemental Material ${ }^{52}$.

Inclusion of $\mathrm{He}$ or $\mathrm{Ne}$ atoms within the alkali oxides and sulfides increases their band gaps. For example, at $2 \mathrm{GPa}$ the band gap of $\mathrm{K}_{2} \mathrm{~S}$ is increased by about $0.4 \mathrm{eV}$ on inclusion of $\mathrm{Ne}$ atoms, and by $1.2 \mathrm{eV}$ for He atoms. The band gap of $\mathrm{Na}_{2} \mathrm{O}$ at $20 \mathrm{GPa}$ is increased by $1.1 \mathrm{eV}$ on including $\mathrm{Ne}$ atoms, but by 3.5 $\mathrm{eV}$ for He atoms. In the cases that we have studied, the band gaps are increased more by including He atoms than Ne atoms. We have investigated the origin of the 
PBE

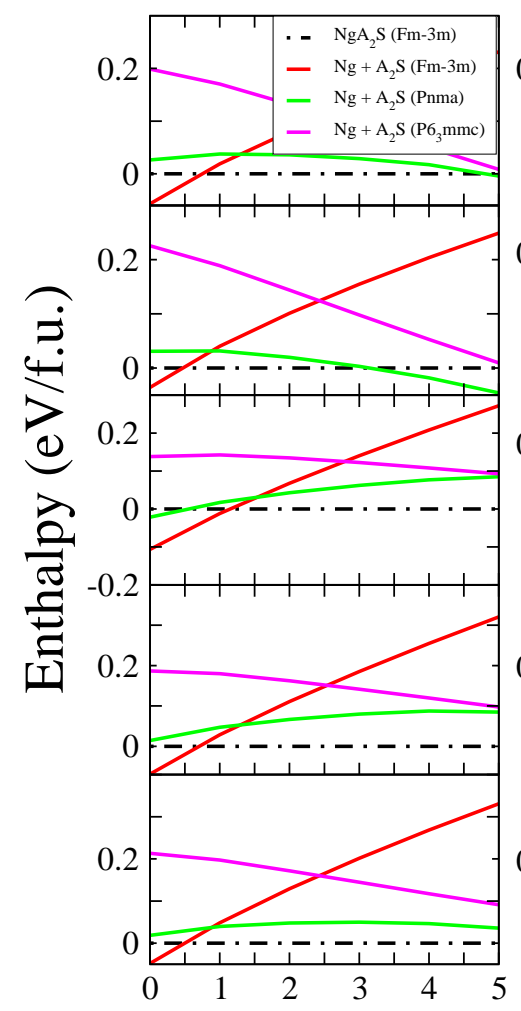

PBEsol

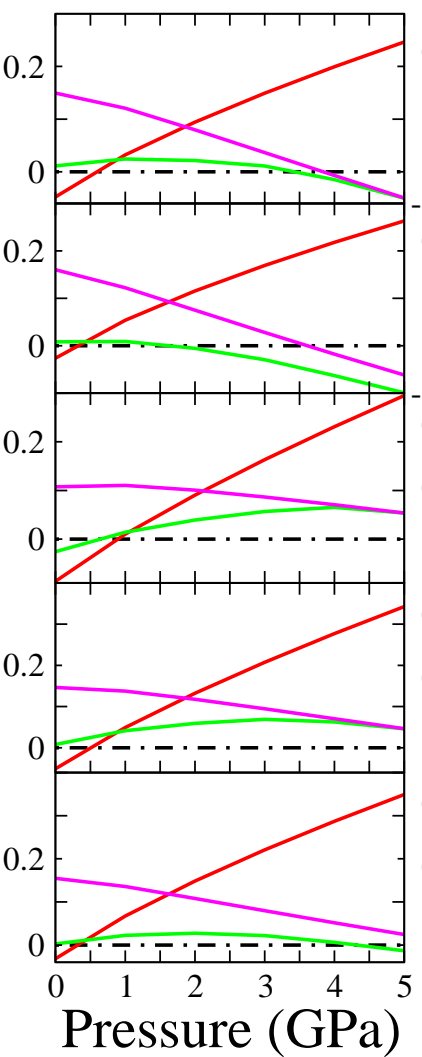

PBE-D3

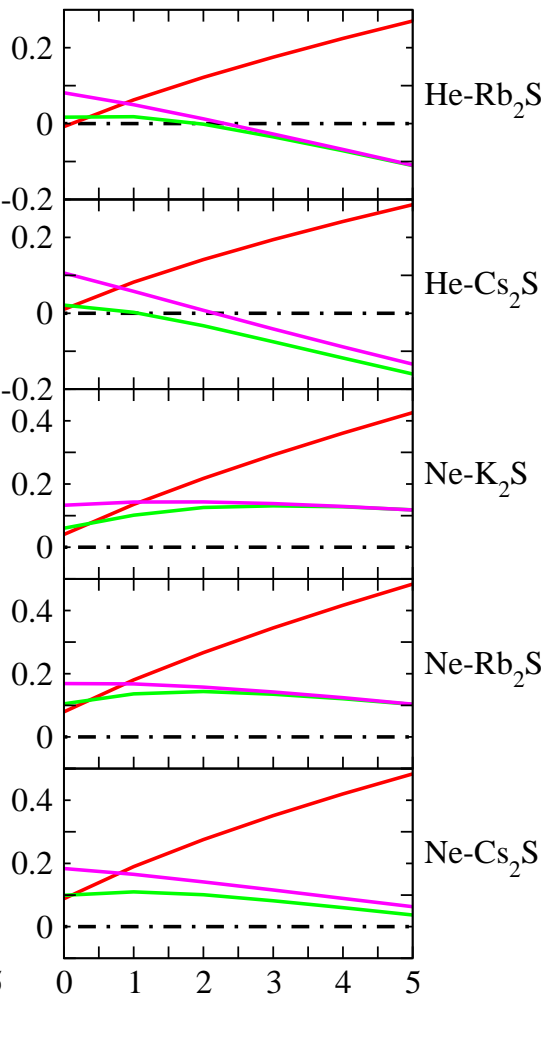

FIG. 3. Enthalpy-pressure relations for noble gas compounds calculated with the PBE, PBEsol and DFT-D3 functionals.

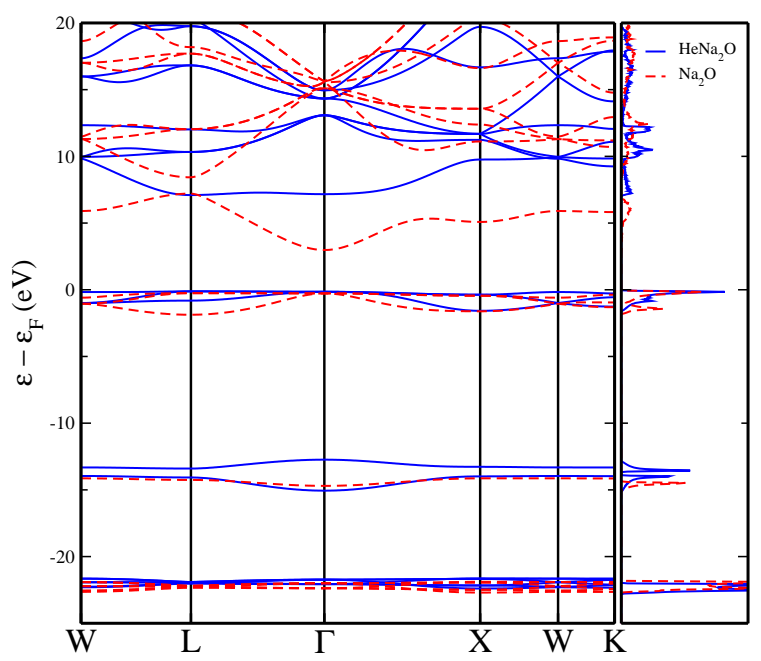

FIG. 4. Electronic Band structure and Density of states of $\mathrm{Na}_{2} \mathrm{O}$ (red dashed line) and $\mathrm{HeNa}_{2} \mathrm{O}$ (blue solid line) at 20 $\mathrm{GPa}$. The zero of energy is at the valence band maximum. The He $1 s$ band lies at about $-13.5 \mathrm{eV}$.

increased band gaps by performing calculations for the oxides and sulfides at the larger volumes of the compounds that include noble gas atoms. We find that the increased gaps are mainly due to the presence of the no- ble gas atoms, rather than the increase in volume. This arises because the nearly-free-electron states (parabolic band around $\Gamma$ point) at the conduction band minimum have a significant weight within the voids of the antifluorite structure, and the presence of the noble gas atoms make the states more localized with almost no dispersion. The band gaps obtained using the PBE functional are a factor of about two smaller than experimental values for $\mathrm{Li}_{2} \mathrm{O}\left(8 \mathrm{eV}^{\underline{53}}\right), \mathrm{Na}_{2} \mathrm{O}\left(4.4-5.8 \mathrm{eV}^{\underline{54}}\right)$, and $\mathrm{K}_{2} \mathrm{O}\left(4.0-5.4 \mathrm{eV}^{54}\right)$. The results from quasiparticle bandstructure calculations within the $G_{0} W_{0}$ approximation ${ }^{55}$ or self-interaction corrections $\frac{56}{}$ are in quite good agreement with the available experimental data. Underestimation of band gaps within standard DFT calculations is well-known, but the changes in band gaps due to the introduction of the noble gas atoms are likely to be significantly more accurate than the gaps themselves. To confirm this conclusion, we have used a HSE06 hybrid functional, which has been successfully applied to Li-ion battery electrode materials 57 to calculate accurate electronic band structures of $\mathrm{Na}_{2} \mathrm{O}$ and $\mathrm{HeNa}_{2} \mathrm{O}$, as shown in the Supplemental Material ${ }^{52}$. Compared with PBE results, both band gaps increase by about $1.8 \mathrm{eV}$ and the changes in band gap caused by inclusion of He are almost same.

The phonon bandstructures of $\mathrm{K}_{2} \mathrm{~S}$ and $\mathrm{NeK}_{2} \mathrm{~S}$ at 5 GPa shown in Fig. 5 indicate that the Ne atoms give rise 
to Einstein-oscillator-like bands at about $110 \mathrm{~cm}^{-1}$. We have investigated the dynamical stability of the $F m \overline{3} m$ structures with incorporated noble gas atoms, finding that the phonon bandstructures of $\mathrm{HeK}_{2} \mathrm{~S}$ and $\mathrm{HeNa}_{2} \mathrm{O}$ have no imaginary frequency modes down to zero pressure, and therefore they might be quench recoverable under ambient conditions.

$\mathrm{HeK}_{2} \mathrm{~S}$ is, however, dynamically unstable at low pressures, but it is stabilized by pressures of a few GPa, see Figs. 6 and 7 of the Supplemental Material ${ }^{52}$. It is neccessary to consider thermal expansion of crystals 58 , and therefore we have corrected the lattice constants and enthalpies of $\mathrm{NeK}_{2} \mathrm{~S}$ using the quasiharmonic approximation (QHA). The lattice parameter changes from $7.04 \AA$ to $7.09 \AA$, with a minor difference of $0.7 \%$. At the static lattice level and a pressure of $5 \mathrm{GPa}, \mathrm{NeK}_{2} \mathrm{~S}$ is about $80 \mathrm{meV} / \mathrm{fu}$ more stable than $\mathrm{Ne}+\mathrm{K}_{2} \mathrm{~S}$, but when quasiharmonic phonon enthalpies are added the stability of $\mathrm{NeK}_{2} \mathrm{~S}$ is reduced to $54 \mathrm{meV} / \mathrm{fu}$. Similarly, at the static lattice level and a pressure of $40 \mathrm{GPa}, \mathrm{HeNa}_{2} \mathrm{O}$ is about $187 \mathrm{meV} / \mathrm{fu}$ more stable than $\mathrm{He}+\mathrm{Na}_{2} \mathrm{O}$. Including vibrational zero-point energy reduces this to $175 \mathrm{meV} / \mathrm{fu}$. We have found strong evidence that materials such as $\mathrm{HeNa}_{2} \mathrm{O}$ and $\mathrm{NeK}_{2} \mathrm{~S}$ could be thermodynamically stable under pressure, and that it might be possible to synthesize them. More information about the phonon bandstructures is provided in the Supplemental Material ${ }^{52}$.

The inclusion of noble gas atoms might be detected via an increase in the lattice constant observable in $\mathrm{x}$ ray diffraction, or in Raman or infrared (IR) vibrational spectroscopy. The antifluorite oxides and sulfides, without noble gas atoms, have one Raman and one IR active vibrational mode. Inclusion of $\mathrm{Ne}$ atoms in $\mathrm{K}_{2} \mathrm{~S}$ at 5 GPa leads to the appearance of an additional vibrational mode at around $110 \mathrm{~cm}^{-1}$, as can be seen in Fig. 5. The Ne-derived normal modes represent the relative motion between $\mathrm{Ne}$ atoms and $\mathrm{K}_{2} \mathrm{~S}$ crystal, as shown in Fig. 5 (b), and the vibrational amplitude of $\mathrm{Ne}$ is much higher than that of $\mathrm{K}$ and $\mathrm{S}$. Other localized high frequency modes are mainly derived from the $\mathrm{S}$ atoms (Fig. 5 (c)(d)). The vibrations are in directions along body diagonals or face diagonals. The zone-center mode of $\mathrm{NeK}_{2} \mathrm{~S}$ at around 110 $\mathrm{cm}^{-1}$ is IR active, and the $\mathrm{NeK}_{2} \mathrm{~S} / \mathrm{K}_{2} \mathrm{~S}$ modes around $150 \mathrm{~cm}^{-1}$ are Raman active, and those around $230 \mathrm{~cm}^{-1}$ are IR active. The calculated Raman and IR intensities for $\mathrm{NeK}_{2} \mathrm{~S} / \mathrm{K}_{2} \mathrm{~S}$ at $5 \mathrm{GPa}$ are shown in the Supplemental Material ${ }^{52}$. The appearance of a new IR active vibrational mode of $\mathrm{NeK}_{2} \mathrm{~S}$ at around $110 \mathrm{~cm}^{-1}$, the shifts in the Raman and IR frequencies, and the changes in Raman intensity could be used as signatures of the inclusion of Ne atoms. Similar data for the inclusion of He within $\mathrm{Na}_{2} \mathrm{O}$ at $40 \mathrm{GPa}$ are reported in the Supplemental Material ${ }^{52}$. As condensed $\mathrm{He}$ or $\mathrm{Ne}$ are commonly used as the pressure transmission medium in diamond-anvilcell experiments 59 , our results are also relevant to the question of whether these atoms could enter alkali oxides and sulfides in diamond-anvil-cell experiments.

\section{CONCLUSION}

In summary, we have predicted the stability of compounds at high pressures in which $\mathrm{He}$ or $\mathrm{Ne}$ atoms are included within alkali metal oxides or sulfides. In some cases structures including noble gas atoms are predicted to become thermodynamically stable at pressures below $1 \mathrm{GPa}$. The chemical interactions between the host oxide or sulfide and the guest noble gas atoms is weak, and the materials discussed here may be described as inclusion compounds. Our study suggests a new class of metal/noble gas inclusion compounds that could be synthesized under applied pressure, and might be useful for gas storage. It seems likely that many more compounds containing noble gas atoms could be stabilized by pressure. We predict that some Ne compounds, such as $\mathrm{NeK}_{2} \mathrm{~S}, \mathrm{NeRb}_{2} \mathrm{~S}$, and $\mathrm{NeCs}_{2} \mathrm{~S}$ might be stable at ambient pressure. We hope that our study may encourage attempts to synthesize this class of compounds.

\section{ACKNOWLEDGMENTS}

The authors thank Tong Chen for the help on HSE06 calculations. J.S. gratefully acknowledges fi-
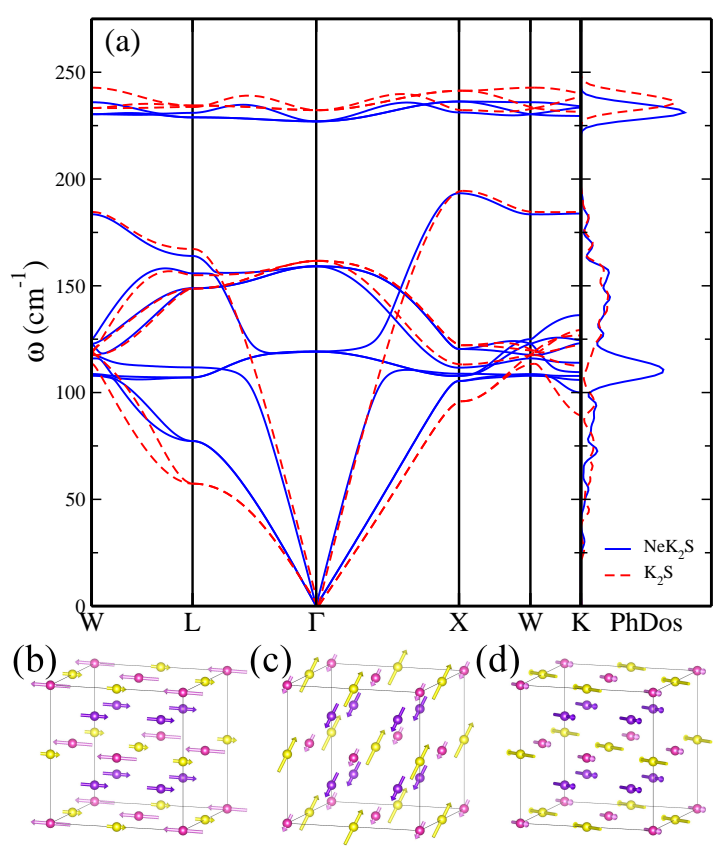

FIG. 5. (a) Phonon bandstructures and densities of states of $\mathrm{K}_{2} \mathrm{~S}$ (red dashed line) and $\mathrm{NeK}_{2} \mathrm{~S}$ (blue solid line) at $5 \mathrm{GPa}$. The Ne-derived vibrations give Einstein-oscillator-like phonon bands at about $110 \mathrm{~cm}^{-1}$ which show some hybridization with the $\mathrm{K}_{2} \mathrm{~S}$-derived bands. (b) The localized modes obtained for Ne at the $\Gamma$ point. (c)(d) The localized modes with highest frequency at the $\Gamma$ point. The vectors in $(b)(c)(d)$ show the polarization directions and amplitudes of the atoms. Ne, K, $\mathrm{S}$ atoms are colored magenta, purple and yellow respectively. 
nancial support from the MOST of China (Grant Nos. 2016YFA0300404, 2015CB921202), the National Natural Science Foundation of China (Grant Nos. 11574133 and 11834006), the NSF of Jiangsu Province (Grant No. BK20150012), Special Program for Applied Research on Super Computation of the NSFC-Guangdong Joint Fund (the second phase) under Grant No. U1501501, the Science Challenge Project (No. TZ2016001), and the Fundamental Research Funds for the Central Universities.
C.J.P. and R.J.N. acknowledge financial support from the Engineering and Physical Sciences Research Council (EPSRC) of the U.K. under grants [EP/G007489/2] (C.J.P.) and [EP/P034616/1] (R.J.N.). C.J.P. also acknowledges financial support from EPSRC and the Royal Society through a Royal Society Wolfson Research Merit award. The calculations were carried out using supercomputers at Nanjing University, "Tianhe-2" at NSCCGuangzhou and the CSD3 Peta4 CPU/KNL machine in the University of Cambridge.
* jiansun@nju.edu.cn

1 H. Tsuji, F. Yagi, and H. Hattori, Chem. Lett., 1881 (1991)
S. Wang, S. Yan, X. Ma, and J. Gong, Energy \& Environmental Science 4, 3805 (2011).

3 U. Martinez, L. Giordano, and G. Pacchioni, J. Chem. Phys. 128, 164707 (2008).

4 E. A. Mikajlo, K. L. Nixon, V. A. Coleman, and M. J. Ford, J. Phys.: Condens. Matter 14, 3587 (2002).

5 E. A. Mikajlo, K. L. Nixon, and M. J. Ford, J. Phys.: Condens. Matter 15, 2155 (2003).

6 E. A. Mikajlo and M. J. Ford,

J. Phys.: Condens. Matter 15, 6955 (2003).

7 K. Kunc, I. Loa, A. Grzechnik, and K. Syassen, Phys. Status Solidi B 242, 1857 (2005).

8 Z̆. Cančarević, J. C. Schön, and M. Jansen, Phys. Rev. B 73, 224114 (2006).

9 A. Lazicki, C.-S. Yoo, W. J. Evans, and W. E. Pickett, Phys. Rev. B 73, 184120 (2006).

10 M. M. Islam, T. Bredow, and C. Minot, J. Phys. Chem. B 110, 9413 (2006).

11 R. D. Eithiraj, G. Jaiganesh, and G. Kalpana, Physica B: Condensed Matter 396, 124 (2007)

12 M. Moakafi, R. Khenata, A. Bouhemadou, H. Khachai, B. Amrani, D. Rached, and M. Rerat, Eur. Phys. J. B 64, 35 (2008)

13 J. Long, L. Yang, Solid State Sciences 19, 12 (2013)

$14 \mathrm{H}$. Sommer R. Hoppe, Z. Anorg. Allg. Chem. 429, 118 (1977)

15 A. Grzechnik, A. Vegas, K. Syassen, I. Loa, M. Hanfland, and M. Jansen, J. Solid State Chem. 154, 603 (2000).

16 A. Vegas, A. Grzechnik, K. Syassen, I. Loa, M. Hanfland, and M. Jansen, Acta Cryst. B 57, 151 (2001).

17 A. Vegas, A. Grzechnik, M. Hanfland, C. Muhle, and M. Jansen, Solid State Sciences 4, PII S1293 (2002)

18 D. Santamaria-Perez, A. Vegas, C. Muehle, and M. Jansen, Acta Cryst. B 67, 109 (2011)

19 D. Santamaria-Perez, A. Vegas, C. Muehle, and M. Jansen, J. Chem. Phys. 135, 054511 (2011).

20 D. Varshney and S. Shriya, Phys. Chem. Minerals 40, 521 (2013)

21 S. Hull, Rep. Prog. Phys. 67, 1233 (2004)

22 W. L. Mao, C. A. Koh, and E. D. Sloan, Phys. Today 60, 42 (2007)

23 D. Londono, W. F. Kuhs, and J. L. Finney, Nature 332, 141 (1988)

24 R. M. Barrer and D. J. Ruzicka, Trans. Faraday Soc. 58, 2239 (1962).
25 P. Teeratchanan and A. Hermann, J. Chem. Phys. 143, 154507 (2015).

26 X. Dong, A. R. Oganov, A. F. Goncharov, E. Stavrou, S. Lobanov, G. Saleh, G.-R. Qian, Q. Zhu, C. Gatti, V. L. Deringer, R. Dronskowski, X.-F. Zhou, V. B. Prakapenka, Z. Konpkov, I. A. Popov, A. I. Boldyrev, and H.-T. Wang, Nat. Chem. 9 (2017).

27 J. A. Sans, F. J. Manjón, C. Popescu, V. P. Cuenca-Gotor, O. Gomis, A. Muñoz, P. RodríguezHernández, J. Contreras-García, J. Pellicer-Porres, A. L. J. Pereira, D. Santamaría-Pérez, and A. Segura, Phys. Rev. B 93, 054102 (2016).

28 Z. Liu, J. Botana, A. Hermann, S. Valdez, E. Zurek, D. Yan, H.-q. Lin, and M.-s. Miao, Nat. Commun. 9, 951 (2018).

29 W. Grochala, Chem. Soc. Rev. 36, 1632 (2007).

30 J. Klimeš, D. R. Bowler, and A. Michaelides, J. Phys.: Condens. Matter 22, 022201 (2010).

31 J. Klimeš, D. R. Bowler, and A. Michaelides, Phys. Rev. B 83, 195131 (2011).

32 K. Lee, É. D. Murray, L. Kong, B. I. Lundqvist, and D. C. Langreth, Phys. Rev. B 82, 081101 (2010).

33 S. Grimme, J. Comput. Chem. 27, 1787 (2006)

34 S. Grimme, J. Antony, S. Ehrlich, and H. Krieg, J. Chem. Phys. 132, 154104 (2010).

35 J. P. Perdew, A. Ruzsinszky, G. I. Csonka, O. A. Vydrov, G. E. Scuseria, L. A. Constantin, X. Zhou, and K. Burke, Phys. Rev. Lett. 100 (2008).

36 P. Touzain, F. Brisse, and M. Caillet, Can. J. Chem. 48, 3358 (1970)

37 A. F. Schuch, E. R. Grilly, and R. L. Mills, Phys. Rev. 110, 775 (1958).

38 D. G. Henshaw, Phys. Rev. 111, 1470 (1958)

39 R. J. Hemley, C. S. Zha, A. P. Jephcoat, H. K. Mao, L. W. Finger, and D. E. Cox, Phys. Rev. B 39, 11820 (1989)

40 S. J. Clark, M. D. Segall, C. J. Pickard, P. J. Hasnip, M. J. Probert, K. Refson, and M. C. Payne, Z. Kristallogr. 220, 567 (2005)

41 C. J. Pickard and R. J. Needs, Phys. Rev. Lett. 97, 045504 (2006).

42 C. J. Pickard and R. J. Needs, J. Phys.: Condens. Matter 23, 053201 (2011).

43 R. J. Needs and C. J. Pickard,

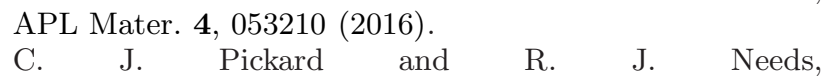
Phys. Rev. Lett. 102, 146401 (2009)

45 J. Sun, D. D. Klug, C. J. Pickard, and R. J. Needs, Phys. Rev. Lett. 106, 145502 (2011) 
${ }^{46}$ K. Xia, J. Sun, C. J. Pickard, D. D. Klug, and R. J. Needs, Phys. Rev. B 95, 144102 (2017).

47 G. Kresse and J. Furthmüller, Comp. Mat. Sci. 6, 15 (1996).

$48 \mathrm{C}$. Cazorla and J.

Boronat, J. Phys.: Condens. Matter 20, 015223 (2008)

49 R. J. Needs, M. D. Towler, N. D. Drummond, and P. L. Ros, J. Phys.: Condens. Matter 22, 023201 (2010)

50 M. Dion, H. Rydberg, E. Schröder, D. C. Langreth, and B. I. Lundqvist, Phys. Rev. Lett. 92, 246401 (2004)

51 J. Heyd, G. E. Scuseria, and M. Ernzerhof, J. Chem. Phys. 118, 8207 (2003)

52 See Supplemental Material at http://link.aps.org/supplemental/.... for more details of the phase transition pressures, electronic bandstruc- tures and densities of states, and phonon bandstructures.

53 Y. Ishii, J. Murakami, and M. Itoh, J. Phys. Soc. Jpn 68, 696 (1999)

54 W. Rauch, Z. Physik 116, 652 (1940)

55 C. Sommer, P. Krüger, and J. Pollmann, Phys. Rev. B 85, 165119 (2012).

56 B. Baumeier, P. Krger, J. Pollmann, and G. V. Vajenine, Phys. Rev. B 78, 125111 (2008).

57 Y. Qi, L. G. Hector, C. James, and K. J. Kim, J. Electrochem. Soc. 161, F3010 (2014).

58 J. Wrbel, L. G. Hector, W. Wolf, S. L. Shang, Z. K. Liu, and K. J. Kurzyd?owski, Journal of Alloys and Compounds 512, 296 (2012)

59 A. Jayaraman, Rev. Mod. Phys. 55, 65 (1983). 